\title{
Archéopages
}

Archéopages

Archéologie et société

Hors-série 1 | 2008

Construction ${ }^{\mathrm{s}}$ de l'archéologie

\section{France et États-Unis : comparaison des institutions archéologiques}

Michael Dietler et Ingrid Herbich

\section{(2) OpenEdition}

1 Journals

Édition électronique

URL : https://journals.openedition.org/archeopages/878

DOI : 10.4000/archeopages.878

ISSN : 2269-9872

Éditeur

INRAP - Institut national de recherches archéologiques préventives

Édition imprimée

Date de publication : 1 février 2008

Pagination : 111-116

ISSN : 1622-8545

\section{Référence électronique}

Michael Dietler et Ingrid Herbich, «France et États-Unis : comparaison des institutions

archéologiques », Archéopages [En ligne], Hors-série 1 | 2008, mis en ligne le 01 février 2008, consulté

le 23 février 2023. URL : http://journals.openedition.org/archeopages/878 ; DOI : https://doi.org/

10.4000 /archeopages. 878 
est ces dernières années en perte de vitesse pour des raisons en grande partie structurelles. Aussi, le comité de rédaction [rénové et réduit] a-t-il décidé de lancer un débat sur la place que [la revue] peut occuper et le contenu qu'elle doit adopter, dans un environnement instable et mouvant». De nouvelles Nouvelles sont en train de naître. Vive les nouvelles Les Nouvelles de l'Archéologie!

Chapelot J., Schnapp A. (dir.) 1984: La Politique de l'archéologie en Europe : actes de la table ronde tenue à Paris les 4 et 5 avril 1978, Paris, Éditions du CNRS.

Cleuziou S., Coudart A., Demoule J.-P., Schnapp A. 1982: «La crise de l'archéologie en France, diagnostic et remèdes», in Godelier M. (dir.), Les Sciences de l'homme et de la société en France, rapports complémentaires, vol. 2, Paris, La Documentation française, p. 40-52.

\section{Construire la recherche à l'Inrap}

Pascal Depaepe

Inrap

la fallu près de trente ans pour que la France se dote d'une structure de recherche

archéologique d'État répondant aux destructions que provoque l'aménagement accéléré du territoire, ${ }^{\mathbf{1}}$ et cela dans un triple souci, patrimonial, scientifique et culturel.

Résultat d'une succession de crises qui s'échelonnent sur près de quinze ans (Bellan 2002), l'adoption de la loi sur l'archéologie préventive en janvier 2001 prévoyait la création d'un établissement public de recherche: ce sera l'Institut national de recherches archéologiques préventives, l'Inrap, qui a été effectivement créé en février 2002 par latransformation du statut de l'Association pour les fouilles archéologiques nationales (Afan).

D'une part héritier de l'Afan, d'autre part jeune institut de recherche, placé sous la double tutelle des ministères chargés de la culture et de la recherche, doté d'un conseil scientifique et d'une direction scientifique et technique (Dst), l'Inrap, après six ans d'existence, construit patiemment et passionnément son identité scientifique. Cette construction se fonde sur deux missions complémentaires: la conduite d'opérations d'archéologie préventive et l'exploitation scientifique et la diffusion de leurs résultats.

Il s'agit donc d'abord et prioritairement d'accomplir les missions opérationnelles, dans le respect des prescriptions de l'État; autrement dit de mener à bien des projets de diagnostics et de fouilles archéologiques. Car cette activité de terrain, conduite sur l'ensemble du territoire national et sur de grandes surfaces, confère à l'Inrap des spécificités scientifiques: elle favorise les approches diachroniques et l'étude spatiale des sites, à l'aide du développement des approches pluridisciplinaires; elle avantage les études comparatives et la définition des ensembles chrono-culturels, de leurs limites et de leurs marges; elle ouvre sur une lecture spatiale des modes de peuplement et permet aussi d'appréhender leurs processus d'évolution.

Cela doit s'ancrer dans une indispensable rigueur méthodologique, mettant l'Inrap en situation de référent en matière de conduite des opérations. C'est pourquoi l'un des axes majeurs de cette construction consiste en l'amélioration et l'harmonisation de la qualité scientifique, qui s'appuie sur l'inventaire et l'analyse des pratiques, des méthodes et des techniques scientifiques. Les séminaires méthodologiques organisés par la direction scientifique et technique, par exemple, sont l'occasion de clarifier les protocoles et procédures existants dans différents domaines - le diagnostic des ensembles funéraires, des sites paléolithiques et mésolithiques, ou encore la chaîne graphique, les inventaires archéologiques...-, d'en faire émerger de nouveaux, de recenser les ressources intellectuelles sur lesquelles peut se fonder l'institut, et de les valoriser en conduisant une réflexion sur les métiers de l'archéologie, leur situations actuelles et leurs évolutions.

Le développement d'une vision de l'occupation des territoires, permise à l'Inrap, passe aussi par une connaissance raisonnée des acquis. Il est donc indispensable d'établir des bilans scientifiques et de doter l'institut d'outils pour y parvenir. Ce point a conduit à la définition d'une politique documentaire, dont l'objet est le recensement, la gestion et la mise à disposition des sources. Premier créateur de données en archéologie, l'Inrap se doit de les valoriser en les mettant à la disposition des différents publics, par le truchement notamment d'un portail scientifique. L'Inrap doit également accentuer sa mission de créateur de connaissances, devenir force de proposition en développant des axes de recherche propres à sa spécificité et renforcer les collaborations avec ses partenaires de la recherche au sein des unités mixtes de recherche.

BELlAN G. 2002: «Archéologie préventive, trois décennies pour une mise en place», Regards sur l'archéologie préventive, Paris, Afan, p.4-13, 57-6o.

\section{France et États-Unis: comparaison des institutions archéologiques}

Michael Dietler, Ingrid Herbich University of Chicago

$\mathbf{N}$ ous souhaitons présenter ici une analyse comparative des paysages institutionnels de l'archéologie en France et aux États-Unis. Le sujet est vaste et complexe, nous ne pourrons donc ici qu'en brosser un rapide portrait. Compte tenu de la dimension de l'archéologie américaine (en nombre d'archéologues, de revues et de publications) et des forces géopolitiques qui interviennent en termes de pouvoir et de symboles 
dans le monde académique international, ce qui relève des États-Unis a inévitablement pour le meilleur et pour le pire-des implications mondiales. Les pratiques américaines ont eu, par exemple, un impact décisif sur le processus de la réforme universitaire européenne, dite «de Bologne», avec des conséquences souvent imprévues.

Pendant des années, nous avons fait la navette - en tant qu'anthropologues et archéologuesentre les systèmes américain et français. Nous en avons retenu l'impression qu'il existait quantité de malentendus, y compris dans les aspects culturels et institutionnels les plus anodins de la pratique universitaire. Il nous a donc paru intéressant de proposer ici une sorte de «traduction culturelle» de ces pratiques.

Le plus souvent, les échanges entre les «écoles de pensées» archéologiques des deux pays concernent les développements théoriques et méthodologiques de la discipline et la différence des approches. Malgré l'importance de ces questions, nous avons souhaité axer notre article sur les contrastes institutionnels, sociaux et culturels qui ont modelé l'archéologie des deux pays - la pratique archéologique ne se résumant évidemment pas à un monde abstrait de confrontations d'idées, de concepts, de théories et de données. Une compréhension profonde des courants théoriques ne peut s'acquérir qu'après avoir compris le paysage institutionnel au sein duquel les acteurs de la discipline agissent et développent leurs problématiques (Dietler 2001).

\section{Départements et disciplines universitaires.}

L'une des différences fondamentales entre les archéologies américaine et française tient au fait que la majorité des archéologues américains reçoivent leur formation au sein de «départements d'anthropologie», l'archéologie étant considérée comme une des quatre sous-disciplines de l'anthropologie. ${ }^{1}$

En France, à l'instar des autres nations européennes, l'archéologie de la Préhistoire récente a été le plus souvent associée à l'histoire, et plus particulièrement à l'histoire nationale. ${ }^{2}$ La raison en est qu'en Europe, cette archéologie s'est professionnalisée dans le contexte de la montée des nationalismes au XIX ${ }^{\mathrm{e}}$ siècle; elle était perçue comme une extension de l'histoire de la nation en quête de d'origines préhistoriques.

Or, au XIX ${ }^{e}$ siècle, les États-Unis ne se prêtaient guère à cette tendancecar les témoins du passé préhistorique appartenaient exclusivement à l'un des peuples dont les cultures étaient alors inconnues des chercheurs (ceux-ci étant, de fait, membres de la société coloniale dominante qui avait, dans un passé récent, décimé les populations indigènes). L'archéologie préhistorique s'est donc essentiellement développée aux côtés de l'ethnographie, pour pratiquer en commun une «ethnographie de sauvetage» et tenter de préserver ce qui apparaissait être les cultures en voie de disparition des premiers Américains.
Un grand nombre de praticiens ont alors utilisé l'ethnographie et l'archéologie pour tenter de restituer les cultures des peuples indigènes, telles qu'elles étaient avant les transformations drastiques dues au colonialisme européen.

Le lien institutionnel et intellectuel qui lie l'archéologie à l'histoire - si familier à la France et aux autres pays européens - se rencontre rarement aux États-Unis. Certes la grande majorité des archéologues universitaires américains sont rattachés à des départements d'anthropologie. Cependant, ceux qui travaillent en Égypte et au Proche-Orient sont souvent regroupés, avec des épigraphistes, des spécialistes des textes anciens ou des historiens d'art, dans des départements de sciences humaines. Ils ont relativement peu de contact et de rapport intellectuel avec les anthropologues. Cette division se reflète dans les deux principales associations professionnelles d'archéologie américaine et leurs publications scientifiques: la Society for American Archaeology (SAA) et sa revue American Antiquity (qui s'adresse surtout aux archéologues anthropologiques), et l'Archaeological Institute of America (AIA) et son American Journal of Archaeology (réservé aux archéologues de l'Antiquité classique et du Proche-Orient).

Même s'il est le résultat accidentel de contingences historiques beaucoup plus larges, le contexte anthropologique de l'archéologie a eu des conséquences intellectuelles importantes pour la discipline. Il a orienté les archéologues américains vers les sciences sociales plutôt que vers les sciences humaines et a exclu, d'emblée, l'étude des cultures comme une fin en soi. Ce contexte a eu également tendance à empêcher que l'archéologie soit conçue comme une discipline distincte (Lenoir, 1997), pour être considérée comme une technique d'investigation au service de problématiques plus larges définies par une vaste communauté de chercheurs. Les archéologues américains ont trois possibilités d'emploi: les universités, les musées et les entreprises CRM (Cultural Resource Management) publiques ou privées. ${ }^{3}$ Il n'y a pas d'institution de recherche équivalant au CNRS français. Dans les trois cas, la majorité des acteurs ont été formés dans des départements d'anthropologie, même si certains musées ont souvent une proportion de chercheurs plus élevée en histoire de l'art ou en Antiquité classique.

Il faut également savoir que, dans la presque totalité des départements d'anthropologie, l'archéologie est une discipline minoritaire, au contraire de l'anthropologie culturelle. Généralement, les archéologues représentent tout au plus 25 pour cent des enseignants d'un département, alors que les anthropologues culturels en constituent au moins la moitié. Ce statut minoritaire détermine les rapports de force à l'intérieur de la discipline anthropologique et influe sur la définition des champs de recherche et sur celle des paradigmes théoriques. Par ailleurs, en dépit du fait que chacun conçoit l'anthropologie comme 
une discipline unifiée, le degré d'intégration varie considérablement d'un département à l'autre, aussi bien intellectuellement que socialement. Dans certains départements, les archéologues et les anthropologues culturels travaillent dans des bâtiments séparés et se rencontrent rarement, sauf à l'occasion de réunions administratives. Dans d'autres, les disciplines sont étroitement imbriquées, avec des cours, des ateliers et des participations mixtes à des comités de thèse, etc. Ces subtiles caractéristiques structurelles ont une influence notable sur le type d'archéologie pratiquée d'un département à l'autre.

\section{L'université américaine et le système}

des colleges. L'une des choses les moins bien comprises par ceux qui ne vivent pas aux États-Unis est le cadre institutionnel qui régit la formation et les recherches des archéologues, à savoir, le système universitaire américain avec ses hiérarchies et ses divisions. Au contraire de la séparation institutionnelle française entre «grandes écoles» (où l'admission se fait sur concours) et universités (où l'admission est libre), les différences de prestige sont, aux États-Unis, institutionnellement moins marquées mais néanmoins très influentes et largement respectées.

La première chose à retenir est la distinction entre les systèmes d'éducation undergraduate et graduate. Un étudiant entame d'abord un cursus undergraduate de quatre années (généralement de 18 à 21 ans), au terme duquel il obtient un diplôme de Bachelor of Arts (BA) ou de Bachelor of Science (BS). Il peut l'acquérir dans un college - une institution dédiée aux études undergraduate qui ne prépare qu'aux BA et BS. Il peut également l'obtenir dans une université qui offre les deux formations: undergraduate et graduate; la seconde conduit au doctorat $(\mathrm{PhD})$. Dans la plupart des cas, l'étudiant obtiendra un diplôme de Master of Arts (MA) au cours de ses études doctorales, à condition d'avoir rempli certaines obligations. Il faut généralement compter huit à dix années d'études après le BA pour l'obtention d'un $\mathrm{PhD}$; ce cycle s'achève avec la soutenance de thèse. Il n'existe pas d'équivalent américain à la nouvelle «habilitation» qui a remplacé, en France, l'ancienne «thèse d'État». L'admission aux études undergraduate et graduate ne se fait qu'après une sélection hautement

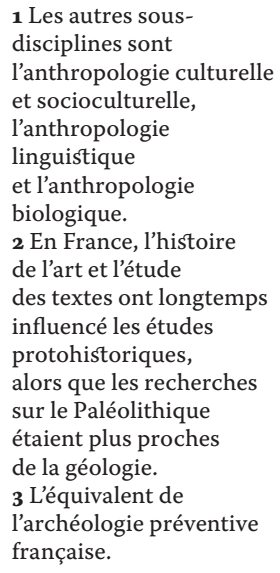

établissements son

national. Le degré

de prestige du cursu

pas forcément lié à celui

jouissent nationalement

d'un prestige élevé

alors qu'ils n'ont pas

de cursus graduate.

En outre, certaine

universités d'État ont

des programmes

alors que leurs du cursus graduate.

graduate très prestigieux, programmes

réputation moyenne.

5 Ce classement est tiré

du National Research

Council, la plus respectée

des organisations qui

publient ce genre

dévaluation. Les chiffres

r les diplômes et

Guide to Anthropolog

Departments,

publié par l'American

Anthropological

Association; et

les données du site

Internet de l'AAA. compétitive. L'étudiant postule aux études doctorales auprès de l'université de son choix, après l'obtention de son BA. Cette université le retiendra sur la base de ses résultats antérieurs, tout en tenant également compte de la réputation de l'institution qu'il l'a formé. ${ }^{*}$

Aux États-Unis, on peut obtenir un BA en anthropologie dans environ 450 colleges et universités, parmi les 1400 établissements d'études supérieures existants, et on peut faire des études doctorales en anthropologies dans 98 universités. En France, il existe environ quinze universités et quatre «grandes écoles» où on peut préparer un diplôme d'études supérieures en archéologie. Aux États-Unis, le nombre moyen de $\mathrm{PhD}$ produits annuellement en anthropologie est relativement stable depuis le milieu des années 1970: autour de 400; la moitié relève de l'anthropologie socioculturelle et 30 pour cent de l'archéologie, ce qui fait environ 120 archéologues diplômés par an. Cependant, à l'instar des institutions undergraduate, la reconnaissance des programmes de doctorat est extrêmement variable; il existe un classement hiérarchique des établissements solidement établi, en fonction de leur prestige. Si on considère les dix départements d'anthropologie les mieux cotés du pays, il apparaît clairement que plus de 60 pour cent des 256 professeurs d'anthropologie de ces établissements sont issus de ces dix mêmes institutions (une grande partie des 40 pour cent restants venant généralement d'universités étrangères renommées). De plus, sept des dix professeurs les plus réputés, appartenant aux dix meilleurs départements, ont été formés dans les quatre départements les mieux classés (University of Chicago, University of Michigan, University of California à Berkeley et Harvard University); le département d'anthropologie de Chicago regroupe 25 pour cent des membres issus de ces quatre institutions (moins de 4 pour cent viennent du département classé en cinquième position ${ }^{5}$ ). On peut épiloguer sur les causes et les significations de ces chiffres, mais ils indiquent clairement que la discipline, au sein de l'anthropologie américaine, se structure autour d'une hiérarchie bien définie, laquelle produit, avec une constance remarquable, une élite qui jouit de l'exclusivité du sommet de l'échelle (Rabinow, 1991).

On constate un phénomène identique à l'intérieur d'un classement plus général d'institutions, parmi lesquelles cinq universités d'élite (Berkeley, Chicago, Harvard, Stanford et Yale) totalisent un nombre exceptionnel de départements parmi les mieux cotés pour un grand nombre de disciplines; elles sont engagées dans un lutte féroce avec les institutions «rivales» en vue d'obtenir le plus grand nombre de départements les mieux classés. Il arrive que, dans une discipline donnée, d'autres universités les dépassent dans le classement, mais aucune ne peut les combattre sur un front aussi étendu (même si certaines n'en sont pas loin). «Combattre» n'est pas un mot trop fort pour 
décrire la relation qui prévaut entre les universités américaines. Elles tentent constamment de détourner les «stars» de l'enseignement, appartenant à d'autres institutions rivales, en leur faisant miroiter toutes sortes de bénéfices, en termes de salaires, de financements de recherche, etc. Tout leur est bon pour améliorer la réputation du département (un peu comme les équipes de football en Europe). En outre, la compétition est permanente entre les meilleurs départements pour attirer les meilleurs graduates (par des offres de bourses de plus en plus alléchantes). Les départements tirent également une grande fierté du succès de leurs étudiants sur le marché national de l'emploi en milieu universitaire; réciproquement, leur succès dans le placement de leurs étudiants à des postes universitaires joue un rôle significatif dans la prise de décision de ces derniers quant au choix du département à rejoindre.

Ce classement national détermine également, pour les universitaires, le rapport entre recherche et enseignement. Dans les établissements les plus prestigieux, la réputation de l'individu est presque exclusivement fondée sur l'impact de ses publications scientifiques; l'enseignement proprement dit joue un rôle relativement mineur dans la sélection d'un professeur ou dans l'attribution d'une tenure (équivalent de la «titularisation» en France). Dans les établissements d'élite, le professeur tend à consacrer moins de temps à son enseignement et plus à sa recherche. En revanche, les professeurs des petits colleges doivent consacrer de longues heures à l'enseignement et beaucoup moins aux publications décisives pour leur promotion.

\section{Universités publiques, universités privées} et centralisation. Une autre différence entre l'archéologie française et l'archéologie américaine réside dans le degré de centralisation et de privatisation des institutions. La plupart des archéologues français - qu'ils soient employés par l'Inrap, une université ou le CNRS - sont des agents de la fonction publique ou assimilés qui opèrent au sein d'administrations très centralisées, souvent coordonnées au niveau national. Le contexte américain fonctionne de façon radicalement différente. Aucune institution nationale n'emploie un nombre important d'archéologues; le recrutement est décentralisé et fortement privatisé. Au contraire de ce qui se passe pour l'Inrap, l'archéologie des CRM relève en grande partie d'entreprises privées qui en tirent profit et rivalisent pour l'obtention des contrats. Aux États-Unis, à part les académies militaires, aucune université n'est subventionnée au niveau national; cependant, chacun des cinquante États subventionne au moins une «université d'État», laquelle contrôle généralement plusieurs campus répartis dans plusieurs villes. En outre, certaines grandes villes (comme New York et Chicago) ont leur propre système de college/université. À côté de ces établissements publics subventionnés et gérés localement par le corps législatif de l'État où ils sont implantés, il existe un nombre important d'universités et de colleges privés qui sont financés par d'importantes fondations, des dons d'anciens élèves et les frais d'inscription et d'étude. Ce sont pour l'essentiel des «sociétés» à but non lucratif, dirigées par un conseil d'administration qui leur est propre, un président et un comité d'enseignants chargés de définir les orientations.

Ces universités privées jouissent d'une grande autonomie. Elles sont généralement prestigieuses et situées au sommet du classement de l'enseignement universitaire. Parmi les cinq universités d'élite citées plus haut, UC Berkeley est la seule université d'État, les quatre autres sont privées. En revanche, quinze des vingt-cinq programmes de $\mathrm{PhD}$ d'anthropologie les mieux cotés relèvent d'une université d'État. Les frais d'inscription et d'étude annuels dans les universités et les colleges privés peuvent s'élever à plus de 35000 dollars, alors que les universités publiques demandent beaucoup moins; un pourcentage élevé d'étudiants des universités privées reçoit cependant une bourse qui réduit de façon substantielle, voire finance entièrement, les frais d'étude $^{\mathbf{6}}$ ). Au niveau undergraduate, la plus grande partie des étudiants des universités d'État viennent de l'État où l'université est située; les établissements privés ont, quant à eux, tendance à recevoir davantage de demandes d'admissions en provenance d'autres États ou d'autres pays. Au niveau graduate, les universités d'État les plus prestigieuses attirent un corps estudiantin national et international.

Les universités publiques tendent également à avoir un pourcentage plus élevé d'étudiants undergraduate que les établissements privés. Par exemple, Berkeley regroupe environ 23000 undergraduates (91 pour cent d'entre eux venant de l'État de Californie) contre environ 9000 graduates (venant du monde entier); quant à l'université (privée) de Chicago, elle compte environ 4400 undergraduates (dont 78 pour cent ne viennent pas de l'État de l'Illinois) contre environ 9000 graduates (venant eux aussi du monde entier ${ }^{7}$ ).

La carrière universitaire. Malgré l'hétérogénéité institutionnelle décrite plus haut, il règne une relative uniformité dans les étapes qui jalonnent une carrière universitaire aux États-Unis, sinon dans les critères qui déterminent l'avancement. Il existe trois niveaux de grade d'enseignants: assistant professor, associate professor et professor (qui ne correspondent pas exactement au système français de maître de conférence et de professeur). On reste habituellement assistant professor pendant sept ans, au terme desquels - à l'issue d'un processus d'évaluation très rigoureux - on obtient une tenure (titularisation). Cette étape marque la transition capitale du grade d'assistant vers celui d'associate professor. Ceux qui n'obtiennent pas la tenure à l'issue de cette réévaluation doivent quitter l'université. Ceux qui l'obtiennent sont assurés 
de la sécurité de l'emploi pour le restant de leurs jours (littéralement, puisqu'il n'y a pas d'âge légal de départ obligatoire à la retraite aux États-Unis). Étant donné la structure hautement démocratique des départements universitaires américains, une fois la tenure obtenue, la différence s'estompe entre associate professor et professor, excepté pour les augmentations graduelles de salaire. Il n'existe pas d'équivalent à la position d'autorité $\mathrm{du}$ «professeur» de la plupart des systèmes universitaires européens. Dans la majorité des universités, la fonction de chef de département (équivalent du directeur d'Ufr français) est temporaire et tournante: il s'agit surtout de représenter le département auprès de l'administration universitaire. On trouve également des spécialistes qualifiés d'instructors ou de lecturers (ce dernier terme n'a rien à voir avec le statut très différent du lecturer des universités britanniques); ils occupent un poste temporaire mal rémunéré qui est dédié à l'enseignement d'une matière. Ils ne font d'ailleurs pas partie du corps enseignant. L'utilisation d'un grand nombre de lecturers est une pratique de plus en plus fréquente dans les universités moins renommées, afin de baisser les coûts; cela a pour effet de créer un système à deux vitesses, probablement préjudiciable aux jeunes chercheurs.

Le financement de l'archéologie. Le financement de la recherche archéologique est un autre élément fondamental de l'archéologie américaine.

Les États-Unis n'ont pas de ministère de la Culture qui prendrait nationalement en charge le financement et l'organisation des fouilles archéologiques. À côté des modestes sommes allouées par les universités, l'archéologie universitaire est principalement financée par des organismes privés ou publics, auxquels on soumet les projets qui sont examinés et financés sur une base concurrentielle. ${ }^{\mathbf{8}}$ La National Science Foundation (NsF) est le plus important de ces organismes; elle est financée par le gouvernement fédéral. La Nsf débourse annuellement environ 12 millions de dollars pour la recherche archéologique et archéométrique (sur un budget total de 4,47 milliards de dollars, toutes sciences confondues); elle finance environ

6 L'admission est

généralement décidée

«en aveugle»:

les étudiants sont

sélectionnés en fonction

de critères académiques,

leur situation financière

restant inconnue.

Une fois retenus,

leur situation financière

est examinée

afin de déterminer

le montant de la bourse

correspondant

à leur besoin.

7 Ces informations

proviennent des sites

internet de

ces universités.

Chicago sort de la norme d'undergraduate

par rapport aux

graduates; la plupart

des autres universités

ont des pourcentages

plus équilibrés,

tandis que les universités

publiques penchent

nettement vers

les undergraduates.

8 Les universités

perçoivent une partie

substantielle

des subventions obtenues

par leur corps enseignant

(bien que les plus forts

montants soient surtout avec son très faible réservés à la physique

La plupart des universités

prélèvent des frais

$50 \%$, voire davantage,

du montant

des subventions obtenues

le coût en est inclus

dans le budget total

de la subvention.
35 pour cent des projets archéologiques qu'elle examine. Mais il existe de nombreuses agences de financement et de sociétés philanthropiques, qui sont des sources potentielles de subventions pour la recherche archéologique.

Quelle que soit l'importance des sommes mises à la disposition de la recherche universitaire, elles ne représentent qu'une modeste fraction de l'argent dépensé par l'archéologie des entreprises de CRM (les entreprises d'archéologie préventive). L'archéologie préventive génère par ailleurs le nombre d'emplois le plus important et l'expansion la plus rapide. Le plus gros des fonds de Crm provient d'entreprises privées contraintes par la loi d'évaluer l'impact environnemental de leurs projets d'aménagement, même si les lois varient énormément d'un État à l'autre.

\section{Départements, spécialités de recherche} et réseaux. La manière dont sont structurés les départements d'anthropologie aux États-Unis conduit rarement à rassembler dans un même département plusieurs spécialistes d'une même région. À quelques exceptions près, la plupart des départements fondent leurs recrutements sur une grande diversité culturelle et géographique, selon la stratégie de représentation dite de «l'arche de Noé». C'est pourquoi on ne compte généralement dans les universités qu'un archéologue spécialisé dans une aire culturelle donnée. Dans les universités d'État, il y a toujours au moins un archéologue de la région, mais ce ne sera pas nécessairement le cas dans les universités privées les plus cotées, lesquelles visent un profil international.

Cette diversité de recrutement a d'importantes conséquences. En premier lieu, il faut travailler avec des collègues et des étudiants qui ne partagent pas le même intérêt régional, mais qui doivent cependant avoir des points communs pour aborder les questions théoriques et les orientations méthodologiques: un étudiant qui consacre sa thèse au Néolithique espagnol ne pourra avoir dans son jury qu'un spécialiste de cette région, les autres seront des archéologues travaillant sur des sujets théoriquement similaires, mais dans d'autres contextes (l'Amérique du Sud ou la Chine, par exemple) ou bien des anthropologues culturels utilisant des concepts qui soient adaptés au sujet de la thèse. Cette situation tend à renforcer le poids du débat théorique par rapport à l'histoire de la culture régionale. Cela implique également d'être constamment confronté aux archives archéologiques de régions différentes de celle de laquelle on est spécialiste et, partant, de renforcer la perspective comparative de ses propres données.

Colloques, réseaux et rituels. Les spécialistes d'une région étant dispersés à travers tout le pays et dans différentes universités, la communication directe a surtout lieu lors des congrès nationaux annuels des principales sociétés américaines d'anthropologie et d'archéologie ou lors de 
colloques thématiques. Ces réunions occupent une place importante dans le paysage institutionnel de l'archéologie américaine, pas simplement en raison du contenu intellectuel des communications qui y sont présentées, mais aussi parce qu'elles offrent aux jeunes chercheurs l'occasion d'entrer sur le marché de l'emploi universitaire, et aux départements de renouveler le corps de leurs enseignants. Elles favorisent la constitution de «réseaux» lors des manifestations socialesqui sont autant d'occasions de discussions, de collaborations, d'échanges d'informations diverses qui contribuent à la vie de la recherche.

Les colloques les plus fréquentés et les plus importants - comme ceux de l'American Anthropological Association (AAA) ou de la Society for American Archaeology (SAA)attirent plusieurs milliers de spécialistes qui viennent de l'ensemble du pays et de l'étranger; ces réunions proposent des milliers de communications présentées dans des centaines de sessions parallèles. Le congrès de 2007 de la SAA, qui s'est tenu à Austin au Texas, a ainsi réuni, sur cinq jours, 3653 participants regroupés en 219 sessions.

Ces congrès nationaux montrent également la relation conflictuelle qui peut parfois exister entre ce que Bourdieu (1984) appelait «le capital académique» et le «capital intellectuel». Comme celui-ci le faisait remarquer, les chercheurs qui investissent lourdement dans l'acquisition du «capital académique» (à savoir le pouvoir administratif qui se développe au sein des départements, universités, organismes scientifiques nationaux, etc.) ne sont pas nécessairement ceux qui possèdent le plus de «capital intellectuel», et vice-versa. C'est pourquoi ces rencontres fonctionnent un peu comme une arène rituelle où s'affrontent ces deux formes de pouvoir et où la concurrence s'étale au grand jour et sur la scène nationale. Mais elles permettent également aux «réseaux» scientifiques dispersés à travers le pays de se reconstruire annuellement en tant que «communauté imaginaire» unifiée de l'archéologie américaine et suscitent un sentiment profond de rassemblement et de renouveau de la communauté.

Conclusion. N’ont été évoqués dans ces lignes que quelques thèmes qu'il nous a semblé important de souligner pour une meilleure compréhension du paysage institutionnel de l'archéologie américaine contemporaine en relation avec la situation française.

C'est un domaine universitaire gigantesque où des milliers de chercheurs conduisent quantité de projets. Nos choix se sont donc ici fatalement limités à notre expérience et à nos intérêts personnels. Néanmoins, nous espérons que cet exercice aura fourni une traduction pratique des mondes complexes de l'archéologie américaine comparée à l'archéologie française. Nous espérons qu'il aidera à situer les débats intellectuels et des thèmes théoriques des recherches car il n'est pas aisé d'acquérir ce type d'informations implicitement partagées mais faisant rarement l'objet d'une publication.

Bourdieu P. 1984 : Homo Academicus. Paris : Éditions de Minuit.

Dietrer M. 2001 : « American archaeology at the Millennium: a user's guide ». Revista d'Arqueologia de Ponent, Universitat de Lleida, 11, p. 7-20.

LENOIR T. 1997: Instituting Science: The Cultural Production of Scientific Disciplines. Stanford : Stanford University Press.

RABINOw P. 1991 : «For hire: resolutely late modern ». Recapturing Anthropology: Working in the Present, edited by R.G. Fox, Santa Fe: SAR Press, p. 59-72.

\section{L'archéologie entre Paris et Rome}

\section{Michel Gras}

École française de Rome

J e voudrais contribuer, à partir de mon observatoire romain, à la nécessaire réflexion permanente sur le métier d'archéologue et sur la politique archéologique, à l'intention de nos décideurs mais aussi de notre milieu. Je le ferai à partir de ma triple expérience, celle d'un ancien directeur adjoint au CNRS, celle d'un ancien vice-président du CNRA et enfin celle d'un témoin attentif depuis trente ans de l'archéologie italienne, aujourd'hui en tant que directeur de l'École française de Rome.

L’observateur impliqué et distancié que je suis, regardant les politiques patrimoniales et notamment archéologiques depuis la ville qui est depuis Raphäel au cœur de la protection du patrimoine, parfois non sans mal, ne peut pas ne pas faire un retour en arrière pour se rappeler que la véritable archéologie, dans les années 1960, semblait à tous, sauf aux préhistoriens, aux protohistoriens et aux médiévistes, celle des glorieux aînés qui avaient travaillé à Rome, à Athènes, à Beyrouth, au Caire. C'était le temps où l'on admirait Delphine Seyrig dans les films de Resnais ou de Truffaut, mais surtout son père, Henri Seyrig, qui souhaitait la création d'un Institut national d'archéologie pour conduire en France une politique cohérente et efficace dans ce domaine. Il y a donc ceux qui, comme moi, ont voulu travailler hors de nos frontières et ceux qui ont voulu «rester au pays»: et je ne peux que constater aujourd'hui que le travail qui, collectivement, a été effectué par ces derniers mérite l'admiration car beaucoup de chemin a été parcouru, même si les recommandations de Seyrig n'ont pas été suivies.

Arrivant à Rome en 1973, je mesurai l'écart qu'il y avait entre une archéologie métropolitaine balbutiante, où le professeur d'archéologie classique chargé de la circonscription des Antiquités (historiques) faisait bénévolement du mieux qu'il pouvait sans aucun moyen, dans le meilleur des cas avec un chercheur du CNRS isolé, et une archéologie italienne où les surintendants m'apparaissaient comme les héritiers directs des princes de la Renaissance, contrôlant totalement leurs territoires et exposant avec brio les nouvelles découvertes: ainsi 